\title{
Pyrolysis kinetic analysis of sequential extract residues from Hefeng sub-bituminous coal based on Coats-Redfern method
}

\section{Xian-Jin Huang}

Wen-Long Mo ( $\nabla$ mowenlong@xju.edu.cn )

Xinjiang University

Ya-Ya Ma

Xinjiang University

Xiao-Qiang He

Xinjiang University

Yelixiati Syls

Xinjiang University

Xian-Yong Wei

China University of Mining and Technology

Xing Fan

Shandong University of Science and Technology

Xiao-Qin Yang

Xinjiang Yihua Chemical Industry Co., Ltd

Shu-Pei Zhang

Xinjiang Yihua Chemical Industry Co., Ltd

\section{Research Article}

Keywords: Extract residues, Pyrolysis, Coats-Redfern method, Kinetic analysis

Posted Date: November 30th, 2021

DOI: https://doi.org/10.21203/rs.3.rs-1109976/v1

License: (c) (i) This work is licensed under a Creative Commons Attribution 4.0 International License.

Read Full License 
1 Pyrolysis kinetic analysis of sequential extract residues from Hefeng

2 subbituminous coal based on Coats-Redfern method

3 Xian-Jin Huang ${ }^{1}$, Wen-Long Mo ${ }^{* 1}$, Ya-Ya Ma ${ }^{1}$, Xiao-Qiang He ${ }^{1}$, Yelixiati·Syls ${ }^{1}$, Xian-Yong

4 Wei $^{* 1,2}$, Xing Fan ${ }^{1,3}$, Xiao-Qin Yang ${ }^{4}$, Shu-Pei Zhang ${ }^{4}$

5 1. State Key Laboratory of Chemistry and Utilization of Carbon-Based Energy

$6 \quad$ Resources and Key Laboratory of Coal Clean Conversion \& Chemical Engineering

7 Process (Xinjiang Uyghur Autonomous Region), College of Chemical Engineering,

8 Xinjiang University, Urumqi, Xinjiang 830046, China.

9 2. Key Laboratory of Coal Processing and Efficient Utilization, Ministry of Education,

China University of Mining \& Technology, Xuzhou 221116, Jiangsu, China.

3. College of Chemical and Biological Engineering, Shandong University of Science and Technology, Qingdao, Shandong 266590, China.

4. Xinjiang Yihua Chemical Industry Co., Ltd., Changji 831700, China.

Abstract: Sequential extract residues $\left(\mathrm{R}_{\mathrm{i}}, \mathrm{i}=1,2,3,4,5\right)$ were obtained from Hefeng acid-washing coal $\left(\mathrm{HF}_{\mathrm{AC}}\right)$ by petroleum ether, carbon disulfide, methanol, acetone and isometric carbon disulfide/acetone mixture, sequentially. Pyrolysis behavior of the samples was carried out using thermogravimetry analysis. Coats-Redfern method with different reaction order was used to analyze the pyrolysis kinetic of each sample, and the kinetic parameters, including correlation coefficient $\left(R^{2}\right)$, activation energy $(E)$, pre-exponential factor $(A)$, were calculated. Results showed that the weight loss of extract residues was higher than $\mathrm{HF}_{\mathrm{AC}}$, and pyrolysis behavior varies greatly for residues, which may be due to unstable structure after extraction. From conversiontemperature $(\alpha-T)$ curves, pyrolysis process was divided into three stages: lowtemperature stage $\left(150-350{ }^{\circ} \mathrm{C}\right)$, medium temperature stage $\left(350-550{ }^{\circ} \mathrm{C}\right)$ and high temperature stage $\left(550-950{ }^{\circ} \mathrm{C}\right)$. And the medium temperature stage made great 
contribution to the process of pyrolysis, which was dominated by depolymerization and decomposition reaction, and the effect of kinetic fitting to this stage is better, with $R^{2}$ higher than 0.95 . Relationship between kinetic parameters and reaction order showed that swelling effect might be an important reason for the discrepancy of $E$ for each sample in the process of pyrolysis. And $\operatorname{Ln}(A)-E$ relationship has a great significance to predict $E$ and the $A$ under higher reaction order.

Key words: Extract residues; Pyrolysis; Coats-Redfern method; Kinetic analysis

Corresponding author: Wen-Long Mo, E-mail: mowenlong@xju.edu.cn

Xian-Yong Wei, E-mail:wei_xianyong@163.com

\section{Introduction}

In recent years, increasing consumption of coal, the main energy source in China, has caused serious environmental pollution problems. However, coal still play a leading role in Chinese primary energy consumption for a long time in the future. Therefore, clean and efficient utilization of coal has become the top priority [1-4]. Solvent extraction under mild conditions can separate organic matter from coal by the principle of similar phase dissolution, without damaging the environment, and the structure and composition characteristics of coal can be reflected by analyzing the molecular structure characteristics of the soluble molecules [5-6]. There are five kinds of intermolecular forces in coal, including entanglement interaction between alkyl and alkyl groups, $\pi-\pi$ interaction between aromatic rings, weak hydrogen bond, strong hydrogen bond and hydrogen bond $/ \pi-\pi$ complex interaction. Different solvent can selectively destroy the intermolecular interaction in low-rank coal [7-9]. Therefore, sequential extraction with different solvent can effectively improve the extraction efficiency of organic matter in coal.

Macro-kinetics is widely used in study of coal pyrolysis characteristics and reaction 
mechanism, which shows high theoretical and practical significance for improving clean and efficient utilization of coal [10-11]. Thermogravimetric analysis (TGA) is a kind of thermal analysis technology, which measures the change of material quality with temperature and time in a controlled environment. This method has a lot of advantages, such as simple pretreatment, no reagent and application [12-13]. By the establishment of kinetic model, pyrolysis process of coal can be quantitatively analyzed, which is convenient to understand the pyrolysis characteristics and mechanism of coal. Many theoretical models, including zero-order empirical model, single reaction model, multi-stage reaction model and distributed activation energy model (DAEM), have been put forward by researchers. In addition, many methods for calculating kinetic parameters are proposed according to the above model, including Coats-Redfern method, Kissinger method, Dzawa method and Doyle method. However, a universal model and method has not been developed due to the complexity and difference of coal structure. Among them, single reaction model, which considered the process of coal pyrolysis as an approximately first-order or norder reaction and can well predict the kinetic parameters, has been widely concerned [14].

Liu et al. [15] investigated the effect of coal particle size on pyrolysis performance by Coats-Redfern method. It was found that the smaller the coal particle size is, the faster the surface heating rate, resulting in higher heat flux and lower activation energy. In addition, with the decrease of particle size, the specific surface area increased significantly, and the active sites increased, which could promote the release of primary pyrolysis products. According to TGA, Geng et al. [16] discussed the behavior of kinetics using Shenmu bituminous coal and its pyrolysis products. It was found that pyrolysis process involved many types of covalent and other chemical 
bonds, resulting that cleavage of each bond need different activation energies. Du et al. [17] reported the pyrolysis kinetics of Shenfu coal, and divided the pyrolysis process into three stages. Low temperature stage was contributed by small molecules of gas and water. In the middle temperature region, a large amount of gas and tar vapors were evaporated, with semi-coke and coke formed in the process. And volume shrinkage could be observed in the high temperature range. Wu et al. [18] used $n$-level single reaction model to analyze the pyrolysis characteristics of Xinjiang bituminous coal. It was found that wonderful effect can be obtained in the violent pyrolysis stage when the reaction order $n=2$, and the reaction order $n=1$ is suitable for the polycondensation and secondary cracking stage.

In this paper, pyrolysis kinetics of Xinjiang Hefeng acid-washing coal (HFAC) and its sequential extract residues from petroleum ether (PE), carbon disulfide (CDS), methanol $(M)$, acetone and isometric acetone/carbon disulfide (IMCDSAM) was discussed under the reaction order of $n=1-5$ with single reaction model by CoatsRedfern method. And the structure and pyrolysis characteristics of the samples were also investigated according to the kinetics parameters.

\section{Experimental}

\subsection{Coal sample}

The used subbituminous coal sample was selected from Hefeng, Xinjiang, China. Table 1 shows the proximate and ultimate analyses of Hefeng coal (HF) and its acidwashing sample by hydrochloric acid and hydrofluoric acid.

It can be seen from Table 1, that HF shows the characteristics of high ash, high volatile matter, high ratio of hydrogen carbon and low sulfur. After acid-washing treatment, the ash content in $\mathrm{HF}_{\mathrm{AC}}$ is greatly reduced, from $21.18 \%$ to $1.84 \%$, with the removal rate high of $91.31 \%$, indicating that acid-washing can effectively remove ash 
in coal, thus we can study the pyrolysis characteristics of organic matter in coal, and the effect of ash can be ignored.

\subsection{Sequential extraction process}

The detailed process of aid-washing can be known from our group previous references $[19,20]$. HFAC was successively extracted with petroleum ether (PE), carbon disulfide (CDS), methanol $(M)$, acetone and isometric acetone/carbon disulfide (IMCDSAM) by ultrasonic assisted five-stage extraction. Residues obtained from each stage were recorded as $\mathrm{R}_{\mathrm{i}}(\mathrm{i}=1,2,3,4,5)$, and sequential extraction process is shown in Fig. 1. The yield, structural characteristics and composition distribution of the extract have been described in the literature [21].

\subsection{Thermogravimetric analysis (TGA)}

With $\mathrm{N}_{2}$ as carrier gas, the pyrolysis behavior of $\mathrm{HF}_{A C}$ and $\mathrm{R}_{\mathrm{i}}(\mathrm{i}=1,2,3,4,5)$ was carried out on a thermogravimetric analyzer SDT-Q600 from TA company. The temperature is raised from ambient to $1000^{\circ} \mathrm{C}$, with heating rate of $10^{\circ} \mathrm{C} / \mathrm{min}$.

\subsection{Pyrolysis kinetics analysis}

The pyrolysis kinetics of each sample was studied by conversion and other parameters. In this experiment, Coats-Redfern method was used to calculate the kinetics parameters for the process of pyrolysis with a single heating rate.

According to Coats-Redfern method, it generally has the following expression: when $n=1$ :

$$
\ln \left[-\frac{\ln (1-\alpha)}{\mathrm{T}^{2}}\right]=\ln \left[\frac{\mathrm{AR}}{\varphi \mathrm{E}}\left(1-\frac{2 \mathrm{RT}}{\mathrm{E}}\right)\right]-\frac{\mathrm{E}}{\mathrm{RT}}
$$

when $n \neq 1$ :

$$
\ln \left[\frac{1-(1-\alpha)^{1-n}}{T^{2}(1-n)}\right]=\ln \left[\frac{\mathrm{AR}}{\varphi \mathrm{E}}\left(1-\frac{2 \mathrm{RT}}{\mathrm{E}}\right)\right]-\frac{\mathrm{E}}{\mathrm{RT}}
$$



$\mathrm{J} /(\mathrm{mol} \cdot \mathrm{K}) ; A$ is the pre-exponential factor, $\mathrm{min}^{-1}$. follows:

$$
\alpha=\frac{\mathrm{m}_{0}-\mathrm{m}_{\mathrm{g}}}{\mathrm{m}_{0}-\mathrm{m}_{\mathrm{f}}}
$$

where $\mathrm{m}_{\mathrm{g}}$ is the mass of sample at a certain time (or temperature), $\mathrm{mg}$; $\mathrm{m}_{0}$ is the initial mass of sample, $\mathrm{mg} ; \mathrm{m}_{\mathrm{f}}$ is the ending mass of sample reaction, $\mathrm{mg}$.

$$
\text { When } n=1 \text {, let } \mathrm{y}=\ln \left[-\frac{\ln (1-\alpha)}{\mathrm{T}^{2}}\right] \text {; when } \mathrm{n} \neq 1 \text {, let } \mathrm{y}=\ln \left[\frac{1-(1-\alpha)^{1-\mathrm{n}}}{\mathrm{T}^{2}(1-\mathrm{n})}\right] \text {; let } \mathrm{x}=\frac{1}{\mathrm{~T}} \text {. }
$$

When $n$ takes different reaction orders and plot $y$ against $x$, and the regression equation is carried out, with the slope of $-E / R$ and the intercept of $\operatorname{Ln}(A R / \varphi E)$. Activation energy and pre-exponential factor of the reaction can be calculated according to the regression equation.

\section{Results and discussion}

\subsection{TG analysis}

Fig. 2 shows TG and DTG profiles of Hefeng acid-washing coal and its extract residues. It can be seen from Fig. 2 (a) that there are significant differences in TG profiles to the samples. The weight loss of $\mathrm{HF}_{\mathrm{AC}}$ is smaller of $41.03 \%$. TG profiles of 
$\mathrm{R}_{1}$ and $\mathrm{R}_{2}$ are basically the same, with weight loss of $54.97 \%$ and $55.02 \%$, respectively. TG profiles of $\mathrm{R}_{3}, \mathrm{R}_{4}$ and $\mathrm{R}_{5}$ are similar, with weight loss of $42.33 \%$, $44.92 \%$ and $42.53 \%$, respectively. When temperature is lower than $350{ }^{\circ} \mathrm{C}$, the weight loss of each sample shows little difference, with the value about $10 \%$. This stage is mainly ascribed to the desorption and decarboxylation of adsorbed water and small molecule gases, and the weight loss accounts for $18 \%-25 \%$ of the total pyrolysis process. In addition, it is not difficult to see that the weight loss of the residues was larger than that of $\mathrm{HF}_{\mathrm{AC}}$, which might be derived from that, after solvent extraction, the molecular structure of coal is unstable and some solvents were remained in the residues. Temperature from $350{ }^{\circ} \mathrm{C}$ to $550{ }^{\circ} \mathrm{C}$ is the main thermal decomposition stage, which may be belonged to depolymerization and decomposition of larger molecular structure in coal, accompanied by polycondensation reaction to generate some gases (methane and its homologues, olefins, water, etc.).

It can be seen from DTG diagram in Fig. 2 (b) that the weight loss rate of $\mathrm{R}_{1}$ and $\mathrm{R}_{2}$ is smaller than that of $\mathrm{HF}_{\mathrm{AC}}$. This may be due to the fact that volatile substances are dissolved into the extractant after primary and secondary solvent extraction, which might be leading to the molecular skeleton structure is more stable. Moreover, some solvents may enter into the pores of coal molecular structure to form surface tension, which makes pyrolysis process more difficult. While the weight loss rate of $R_{3}, R_{4}$ and $\mathrm{R}_{5}$, from DTG profiles, is larger than that of $\mathrm{HF}_{\mathrm{AC}}$, which might be due to the influence of swelling on the molecular structure of coal after many times solvent extraction, and the volume expansion might be taken place to accelerate the process of pyrolysis [22]. When temperature is higher than $550{ }^{\circ} \mathrm{C}$, aromatic substances condense, and aromatic nuclei increase, and semi-coke forms coke rock. The weight loss of $R_{1}$ and $R_{2}$ is the largest in this stage. This may be due to the polycondensation 
reaction between residual solvents and substances in the residues, releasing a large amount of small molecular gases. The reason for the small weight loss of $\mathrm{R}_{3}, \mathrm{R}_{4}$ and $\mathrm{R}_{5}$ might be concluded that the macromolecular channels formed by swelling under high temperature rapidly collapse and agglomerate to produce molecules.

It can also be seen from the DTG profiles that there are two weight loss rate peaks. The first weight loss rate peak appeared at about $100{ }^{\circ} \mathrm{C}$, corresponding to the evaporation of water and small molecular gases; the second weight loss rate peak is larger, appearing in the between of $400-500{ }^{\circ} \mathrm{C}$. This range is the main stage of the pyrolysis reaction, and more substances in the sample were depolymerized and small molecular gases were released. It can be seen that the peak value of weight loss rate of $\mathrm{R}_{1}$ and $\mathrm{R}_{2}$ is small of $-0.14 \% \cdot{ }^{\circ} \mathrm{C}^{-1}$. The peak value of weight loss rate of $\mathrm{R}_{3}, \mathrm{R}_{4}, \mathrm{R}_{5}$ and $\mathrm{HF}_{\mathrm{AC}}$ are almost the same, and that of $\mathrm{R}_{4}$ is larger of $-0.22 \% \cdot{ }^{\circ} \mathrm{C}^{-1}$.

\subsection{Conversion-temperature diagram}

Fig. 3 (a) shows the relationship between pyrolysis conversion and temperature of Hefeng acid-washing coal and the corresponding extract residues. It can be seen that there is little difference for the conversion-temperature profiles of the acid-washing coal and its extract residues. Combined TG-DTG profiles with $\alpha-T$ diagram, pyrolysis process can be divided into three stages [23-25], as shown in Fig. 3 (b). The samples contain a small amount of moisture, so the weight loss below $150{ }^{\circ} \mathrm{C}$ is taken as the initial pyrolysis process. The intersection points A of tangent line of TG and DTG profile is taken as the dividing temperature point of the first stage $\left(\mathrm{S} 1,150-350{ }^{\circ} \mathrm{C}\right)$ and $\mathrm{B}$ as the second stage $\left(\mathrm{S} 2,350-550{ }^{\circ} \mathrm{C}\right)$, and the third stage (S3) is ranged from 550-950 ${ }^{\circ} \mathrm{C}$. It can be seen from Fig. 3 (a) that the change trend of conversion profiles is basically consistent in the stage of $150-350{ }^{\circ} \mathrm{C}$. And the conversions of extract residues are higher than that of acid-washing coal, which may be due to the 
desorption of more small molecules in this stage (Fig. 2 (a)). In the stage of 350-550

${ }^{\circ} \mathrm{C}$, the conversions increase rapidly, which may be due to that this temperature region is the main stage of pyrolysis reaction, and a large number of substances are depolymerized and decomposed. Moreover, the conversion profiles are divergent in this temperature range, that is the increasing trend of the conversion of $R_{3}, R_{4}, R_{5}$ and $\mathrm{HF}_{\mathrm{AC}}$ is more obvious. In the stage of $550-950{ }^{\circ} \mathrm{C}$, the conversion of $\mathrm{R}_{3}, \mathrm{R}_{4}, \mathrm{R}_{5}$ and $\mathrm{HF}_{\mathrm{AC}}$ increases slowly, indicating that the pyrolysis process becomes gentle. The result may be caused from that the main reaction in this stage is polycondensation, which is difficult to take place.

\subsection{Coats-Redfern kinetic analysis}

In order to discuss the pyrolysis kinetic characteristics of the samples, kinetic fitting of each sample in the temperature range of $350-550{ }^{\circ} \mathrm{C}$ was carried out by Coats-Redfern method, and the fitting diagram of reaction order $n=0.5-5$ was obtained, as shown in Fig. 4. It can be seen that each reaction order has little effect on $\mathrm{R}_{1}$ and $\mathrm{R}_{2}$, which may be due to the samples have a stable and similar main structure. And our previous work [19] showed that extraction process has an effective effect to organic matter, while does not change the main structure of coal. The fitting curves of $\mathrm{R}_{3}, \mathrm{R}_{4}, \mathrm{R}_{5}$ and $\mathrm{HF}_{\mathrm{AC}}$ have a large deviation when $n=3$, indicating that different reaction order can obviously affect the fitting effect. And this phenomenon needs to be further explored from the pyrolysis reaction mechanism.

\subsection{Calculation of kinetic parameters} equation $(y)$, correlation coefficient $\left(R^{2}\right)$, activation energy $(E)$, pre-exponential factor $(A)$, were calculated under different reaction orders by Coats-Redfern method. Results were shown in Table 2. 
It can be seen that the values of $R^{2}$ are above 0.9500 , indicating that the fitting correlation of each sample is better. Activation energy can reflect the difficulty of pyrolysis reaction. It is not difficult to see that the variation for activation energy is very large after extraction. Residues had lower activation energy than $\mathrm{HF}_{\mathrm{AC}}$, indicating that coal after extraction more conducive to pyrolysis. Pre-exponential factor is a constant determined by the nature of the reaction. The value of a varies greatly in different reaction order. Many studies [25-27] have found that the preexponential factor has great difference under different reaction conditions. The value of $A$ increases with the increase of heating rate and reaction order, and it is also found that there is a "compensation effect" between activation energy and pre-exponential factor. Coats-Redfern integral formula assumes that the term $E / R T$ is far greater than 1 , that is, $2 R T / E \rightarrow 0$. Therefore, the smaller the $2 R T / E$ value, the better the calculation results of kinetic parameters can meet the preconditions of the formula.

\subsection{Relationship between kinetic parameters and reaction order}

Pyrolysis is a macroscopic process, and kinetic parameters can reflect the comprehensive performance of pyrolysis process. The higher the reaction order, the more complex the pyrolysis process. The relationship between kinetic parameters and reaction order in the temperature range of $350-550{ }^{\circ} \mathrm{C}$ is shown in Fig. 5.

Fig. 5(a) shows the relationship between activation energy and reaction order. It can be seen from Fig. 5(a) that activation energy increases with the increases of reaction order for each sample, indicating that the higher the reaction order, the more complex the pyrolysis reaction might be. While activation energy presents significant difference for $\mathrm{HF}_{\mathrm{AC}}$ and its residues, which could be related to the composition difference of each sample after sequential extraction. Ma et al. [28] found that coal sample showed swelling effect in solvent, which is a process of gradual expansion, 
micro-explosion, pore collapse and formation of fragments, and swelling coefficient with different solvent varies greatly. After the process of ultrasonic-assisted sequential extraction, with pore structure of coal sample collapse, with weak bridge bond destroyed and cross-linked network structure slacked, the extract residues could be swelled under different solvents. Therefore, swelling effect might be an important reason for the discrepancy of activation energy for each sample in the process of pyrolysis.

Fig. 5 (b) shows the relationship between correlation coefficient $R^{2}$ and reaction order. The values of $R^{2}$ were all above 0.95 , indicating that the correlation effect was acceptable. Correlation coefficient $R^{2}$ increases firstly and then decreases with the reaction order, and the value of $\mathrm{HF}_{\mathrm{AC}}$ reaches the maximum of 0.972 when the reaction order $n=2$, while the values of all the residues reach the maximum when reaction order $n=1.5$, indicating that kinetic parameters show more accuracy when reaction order $n=1.5-2$.

Fig. 5 (c) shows the relationship between $2 R T / E$ and reaction order. $2 R T / E \rightarrow 0$ is the precondition for the calculation of kinetic parameters for Coats-Redfern method. The lower the value, the higher the reliability of the model is. It can be seen that the value of $2 R T / E$ decreases with the increase of reaction order, indicating that high reaction order can better meet the condition of Coats-Redfern method.

Literatures ${ }^{[29-31]}$ found that pre-exponential factor increases when activation energy increases, and the factor decreases when the activation energy decreases, which is called "dynamic compensation effect". Meng et al. ${ }^{[32]}$ also discovered that there was "dynamic compensation effect" between activation energy and pre-exponential factor in the process of coal gasification, and the gas diffusion has a significant impact on the compensation effect. 
The "dynamic compensation effect" can be expressed by mathematical formula as follows:

$$
\operatorname{Ln}(A)=\mathrm{q} E+\mathrm{p}^{[33]}
$$

Where $q$ and $p$ are the compensation parameters.

In order to further explore the compensation effect of activation energy and preexponential factor, the diagram of $\operatorname{Ln}(A)-E$ is plotted, and the profiles are shown in Fig. 5(d). It can be found that the relationship between activation energy and preexponential factor is almost a line. And there is a one-to-one correspondence between the activation energy and the reaction order, which could be used to predict the activation energy and the pre-exponential factor under higher reaction order.

\section{Conclusions}

In this paper, thermogravimetric analyses of acid-washing coal and its extract residues were carried out. Results showed that the weight losses of all stages for extract residues were higher than that of $\mathrm{HF}_{\mathrm{AC}}$. Coats-Redfern method was used to fit the pyrolysis kinetics under the reaction order of $n=0.5-5$ at different temperature stages, and the kinetic parameters were calculated according to the fitting curves. Results showed that, in the medium temperature range, the correlation coefficient $R^{2}$ is larger, and the fitting effect is better. Relationship between kinetic parameters and reaction order showed that swelling effect might be an important reason for the discrepancy of activation energy for each sample in the process of pyrolysis, and that high reaction order can better meet the condition of Coats-Redfern method. And $\operatorname{Ln}(A)-E$ relationship can be used to predict the activation energy and the preexponential factor under higher reaction order.

\section{Acknowledgements}

This work was supported by the Tianchi project for introducing high-level talents to 
Xinjiang Uyghur Autonomous Region (China), Key Laboratory of Coal Processing and Efficient Utilization from Ministry of Education and Open Project of Key Laboratory of Xinjiang Uygur Autonomous Region (Grant 2018D04008).

\section{References}

[1] Tahmasebi A, Yu J, Han Y, Yin F, Bhattacharya S, Stokie D. Study of chemical structure changes of Chinese lignite upon drying in superheated steam, microwave, and hot air[J]. Energy Fuels, 2012, 26(6):3651-60.

[2] Kong J, Wei XY, Li ZK, Yan HL, Zhao MX, Zong ZM. Identification of organonitrogen and organooxygen compounds in the extraction residue from Buliangou subbituminous coal by FTICRMS[J]. Fuel, 2016, 171:151-158.

[3] Liu FJ, Zong ZM, Li WT, et al. A three-step dissociation method for converting Xiaolongtan lignite into soluble organic compounds: Insights into chemicals, geochemical clues, and structural characteristics[J]. Fuel, 2018,242(242):883-892.

[4] He XQ, Mo WL, Wang Q, Ma YY, Ma FY, Fan X, Wei XY. Effect of swelling treatment by organic solvent on the structure and pyrolysis performance of the direct coal liquefaction residue[J]. Energy \& Fuels, 2020, 34(7): 8685-8696.

[5] Lei Z, Zhang Y, Wu L, et al. The dissolution of lignite in ionic liquids[J]. RSC Advances, 2013, 3(7): 2385-2389.

[6] Liu F J, Wei X Y, Xie R L, Wang Y G, Li W T, Li Z K, et al. Characterization of oxygencontaining species in methanolysis products of the extraction residue from Xianfeng lignite with negative-ion electrospray ionization Fourier transform ion cyclotron resonance mass spectrometry[J]. Energy Fuels, 2014, 28:5596-605.

[7] Hu R N, Wang Z C, Li L, Wang X L, Shui H F, et al. Effect of solvent extraction pretreatments on the variation of macromolecular structure of low rank coals $[\mathrm{J}]$. Journal of Fuel Chemistry and Technology, 2018, 46(7): 778-786. 
[8] Lv JH, Wei XY, Wang YH, et al. Mass spectrometric analyses of biomarkers and oxygen-containing species in petroleum ether-extractable portions from two Chinese coals[J]. Fuel, 2016, 173:260-267.

[9] Shui H, Zhou Y, Li H, et al. Thermal dissolution of Shenfu coal in different solvents[J]. Fuel, 2013, 108:385-390.

[10] Heydari M, Rahman M, Gupta R. Kinetic study and thermal decomposition behavior of lignite coal [J]. International Journal of Chemical Engineering, 2015,1-9. [11] Song HJ, Liu GR, Zhang JZ, et al. Pyrolysis characteristics and kinetics of low rank coals by TG-FTIR method [J]. Fuel Processing Technology, 2017, 156: 454-460. [12] Guo YH, Cheng F. Adaptability Analysis of Kinetic Model of Blended Coal Pyrolysis[J]. Journal of Combustion Science and Technology, 2019, 25(6): 509-518.

[13] $\mathrm{Wu} \mathrm{D,} \mathrm{Liu} \mathrm{GJ,} \mathrm{Sun} \mathrm{RY.} \mathrm{Investigation} \mathrm{on} \mathrm{structural} \mathrm{and} \mathrm{thermodynamic}$ characteristics of perhydrous bituminous coal by Fourier transform infrared spectroscopy and thermogravimetry/mass spectrometry[J]. Energy Fuels 2014, 28:3024-3035.

[14] W. de Jong, G. Di Nola, B.C.H. Venneker, H. Spliethoff, M.A. Wójtowicz. TGFTIR pyrolysis of coal and secondary biomass fuels: determination of pyrolysis kinetic parameters for main species and $\mathrm{NO}_{\mathrm{x}}$ precursors[J]. Fuel, 2007, 86(15):23672376.

[15] Liu JX, Ma JF, Luo L, et al. Pyrolysis of superfine pulverized coal. part 5. thermogravimetric analysis[J]. Energy Conversion and Management, 2017, 154:491502.

[16] Geng CC, SY Li, Yue CT, Ma Y. Pyrolysis characteristics of bituminous coal[J]. Elsevier Ltd,2016,89(4):1-6.

[17] Du RL, Wu K, Zhang L, et al. Thermal behavior and kinetic study on the 
pyrolysis of Shenfu coal by sectioning method[J]. Journal of Thermal Analysis and Calorimetry; 2016, 125(2):959-966.

[18] Wu F, Wang MH. Research on the pyrolysis characteristic and kinetics of Xinjiang bituminous coals[J]. China Coal, 2019,45(02):84-90+95.

[19] Ma YY, Ma FY, Mo WL, Wang Q. Five-stage sequential extraction of Hefeng coal and direct liquefaction performance of the extraction residue[J]. Fuel, 2020, 266, 117039-117048.

[20] Wang Y, Ma YY, Mo WL, Gong WT, Ma FY, Fan X, Wei XY, Zhang SP. Functional groups of sequential extracts and corresponding residues from Hefeng sub-bituminous coal based on FT-IR analysis[J]. Journal of Fuel Chemistry and Technology ,2021, 21, 1-12.

[21] Sarwar A, Khan M N, Azhar K F. Kinetic studies of pyrolysis and combustion of Thar coal by thermogravimetry and chemometric data analysis[J]. Journal of Thermal Analysis and Calorimetry, 2012, 109(1): 97-103.

[22] Ma YY, Wang HH, Mo WL, Zhang XY, et al. Effect of swelling by organic solvent on structure, pyrolysis and methanol extraction performance of Hefeng bituminous Coal [J]. ACS Omega, 2021, 6(23): 14765-14773.

[23] Liu QH, Wu K, Du RL, She Y, Liu X. Kinetic analysis of tar's separation from lump Coal[J]. The Iron and Steel Institute of Japan, 2015, 55(5):947-951.

[24] Wang T, Li C, Zhou BX, et al. Experimental investigation of thermal effect in coal pyrolysis process[J]. Fuel Processing Technology, 2020, 200:106269.

[25] Wang YP, Zhang YF, Zhou Q, et al. Thermal kinetics analysis of coal-gangue selected from Inner Mongolia in China[J]. Journal of Thermal Analysis and Calorimetry, 2018, 131(2):1835-1843.

[26] Lv S, Lv GJ, Jiang XG, et al. Pyrolysis/combustion characteristics and kinetic 
analysis of Indonesia lignite sludge[J]. Journal of China Coal Society, 2014, 39(3): $554-561$.

[27] Ankit A. Jain, Anurag Mehra, Vivek V. Ranade. Processing of TGA data: analysis of isoconversional and model fitting methods[J]. Fuel, 2016, 165:490-498.

[28] Ma YY, Ma Fy, He F, et al. Influence of microwave swelling with cavitated creosote oil on the direct liquefaction performance of Xigou coal from Xinjiang and its dynamics analysis[J]. Journal of China Coal Society, 2017, 42(10): 2733-2741.

[29] Czajka K, Kisiela A, Moroń W, Ferens W, Rybak W. Pyrolysis of solid fuels: thermochemical behaviour, kinetics and compensation effect[J]. Fuel Processing Technology, 2016,142:42-53.

[30] Skodras G, Nenes G, Zafeiriou N, Low rank coal- $\mathrm{CO}_{2}$ gasification: experimental study, analysis of the kinetic parameters by Weibull distribution and compensation effect[J]. Applied Thermal Engineering, 74 (2015), 111-118.

[31] Marbán G, Laura Río. Tentative explanation for the kinetic compensation effect in doped catalysts[J]. Kinetics and Catalysis, 2013, 54(4):487-492.

[32] Meng DX, Wang T, Xu JL, Chen XL. Diffusion effect and evolution of kinetic parameters during coal char- $\mathrm{CO}_{2}$ gasification[J]. Fuel, 2019, 255, 115819.

[33] Rasam S, Haghighi A M, Kolsoom A, et al. Thermal behavior, thermodynamics and kinetics of co-pyrolysis of binary and ternary mixtures of biomass through thermogravimetric analysis[J]. Fuel, 2020, 280, 118665. 
Figures

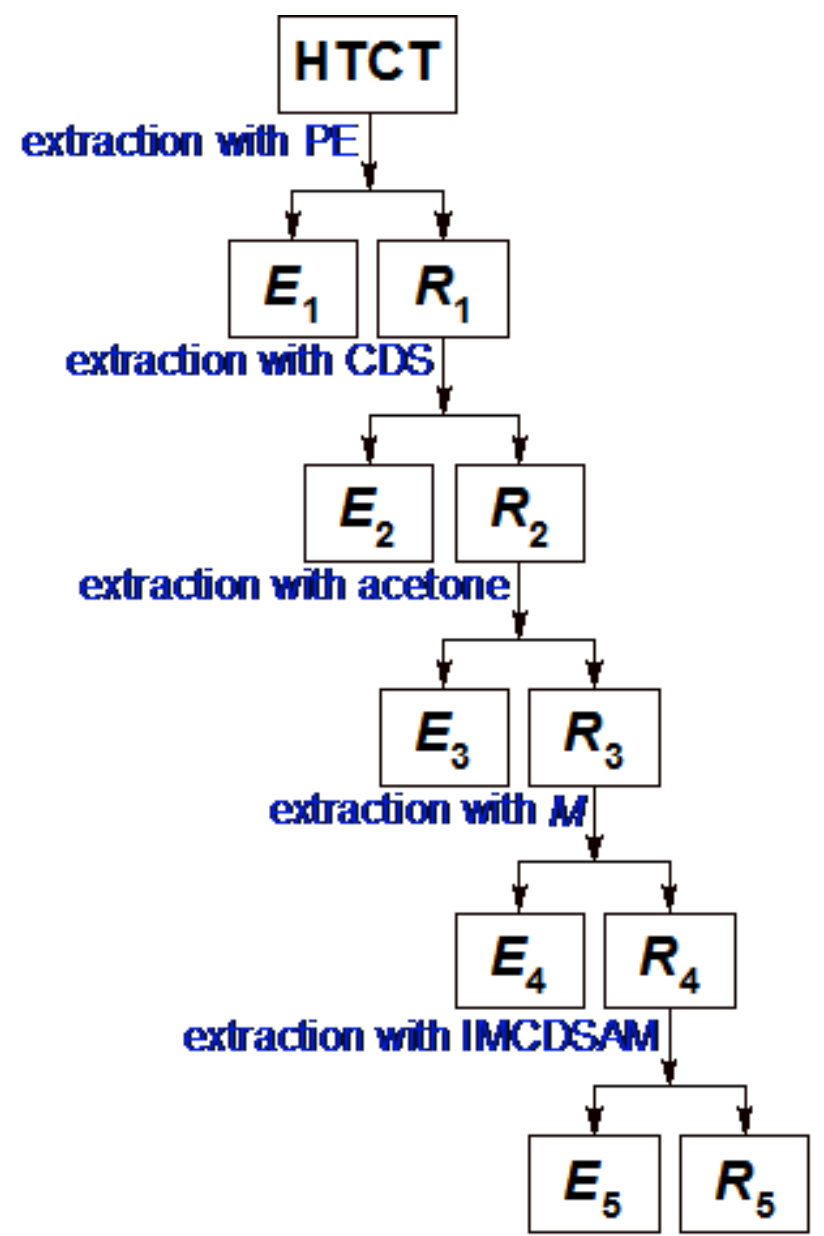

\section{Figure 1}

Sequential extraction process of HFAC.
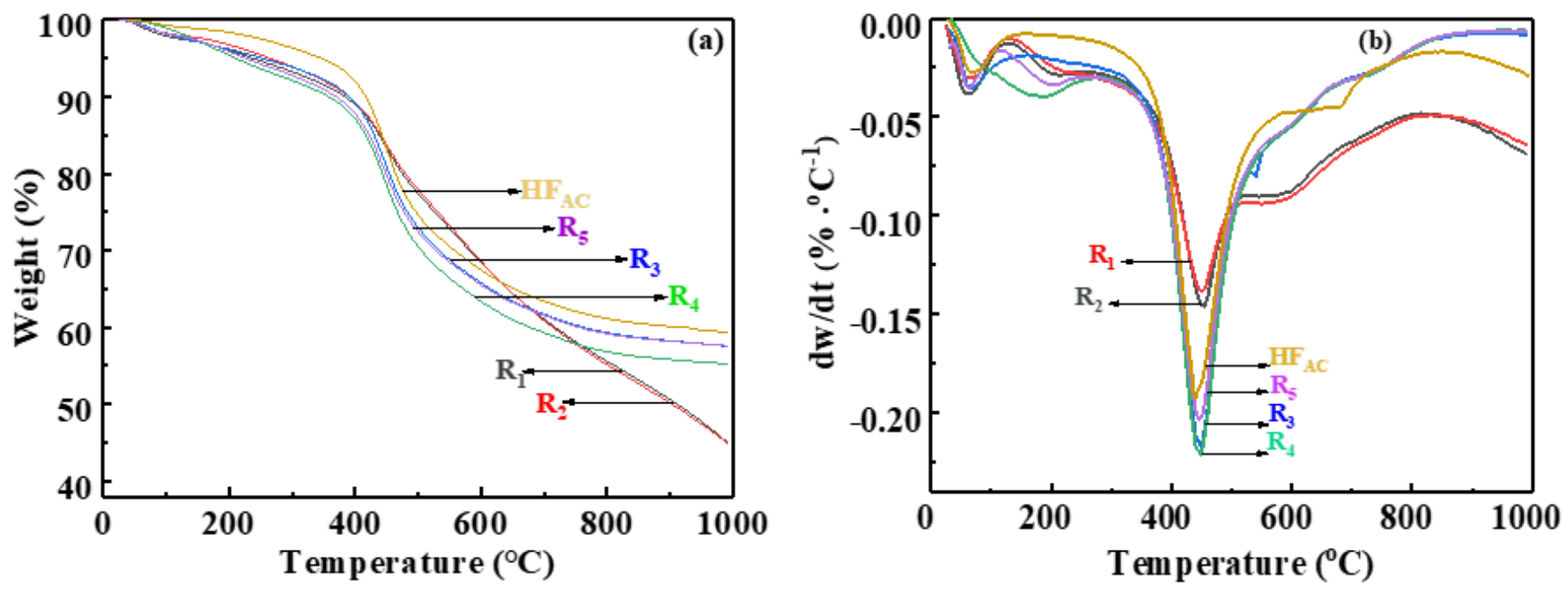
Figure 2

TG-DTG diagram of HFAC and its extract residues
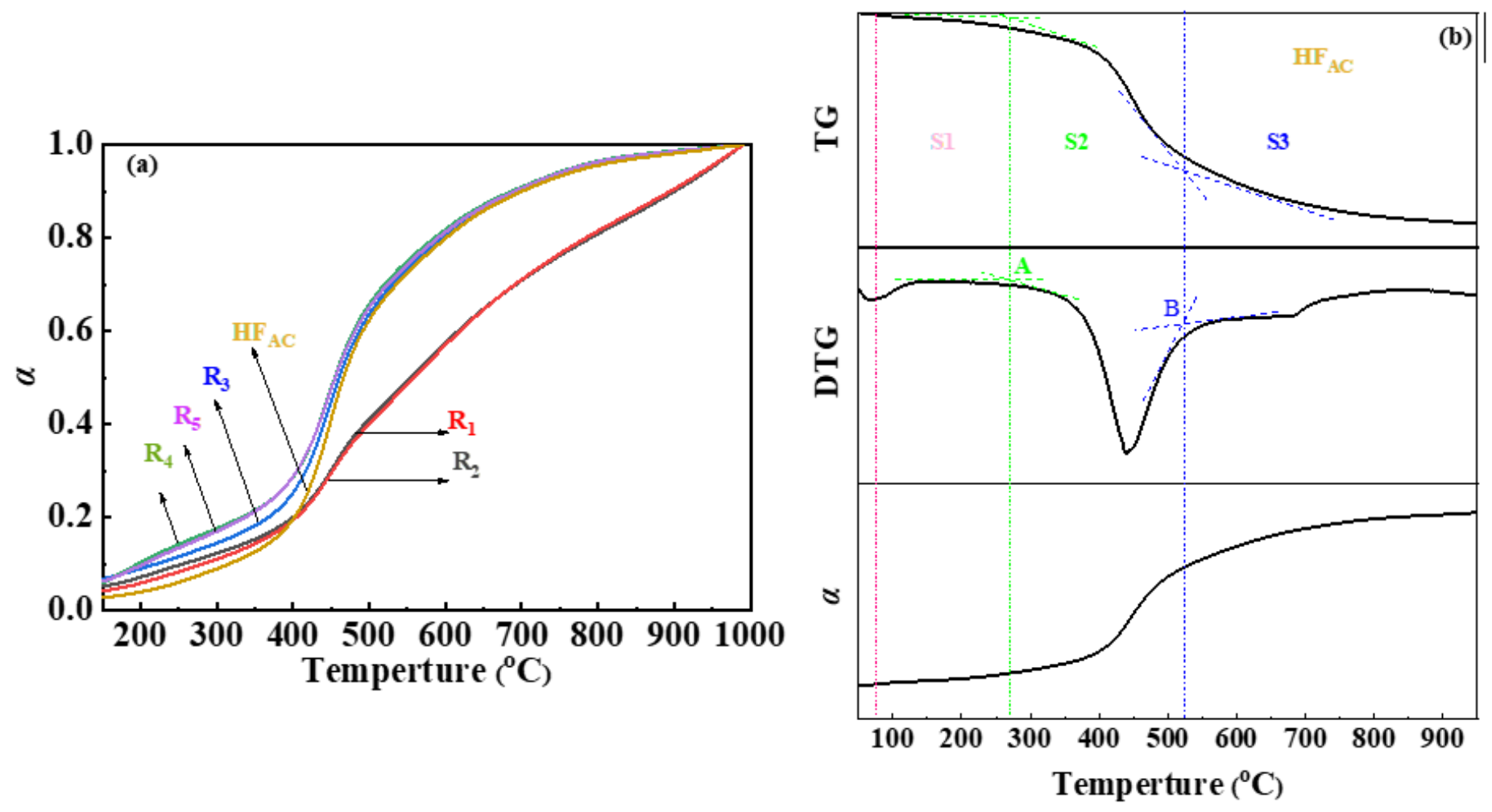

Figure 3

Diagram of conversion to temperature. (a): a-T ; (b): TG/DTG/a-T 

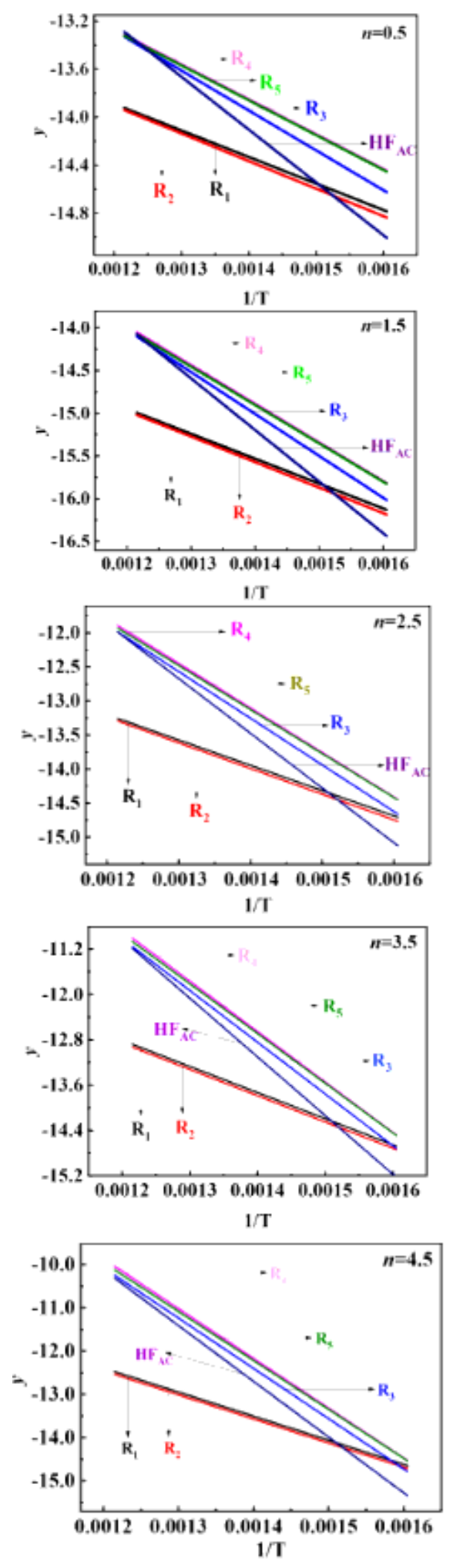

\section{Figure 4}

Fitting curves of each sample with reaction order $n=0.5-5$.
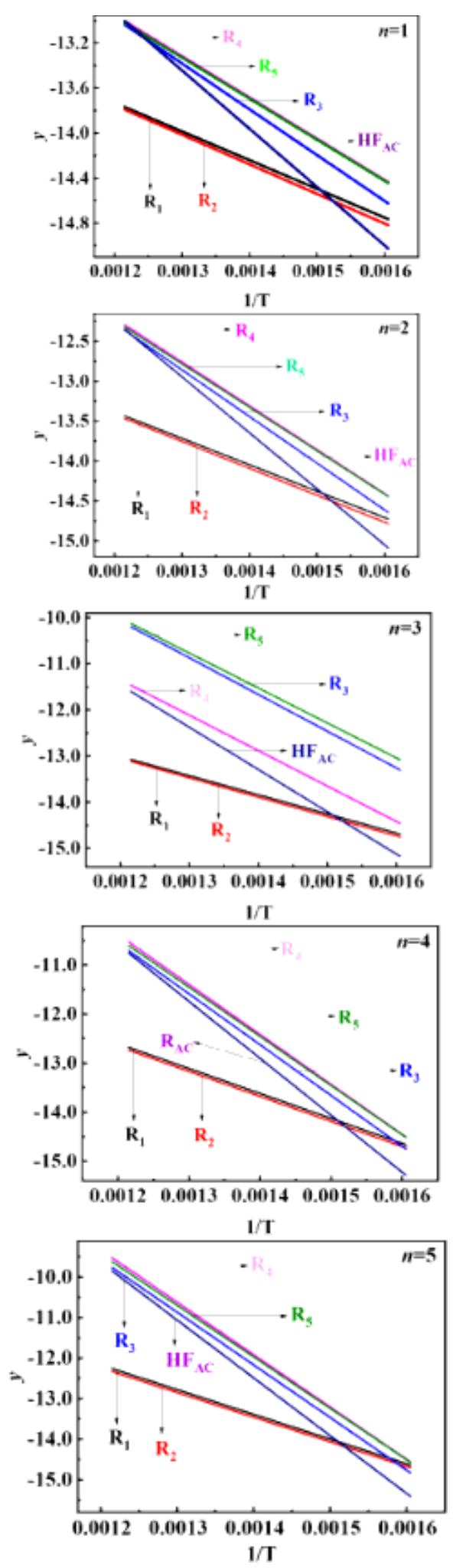

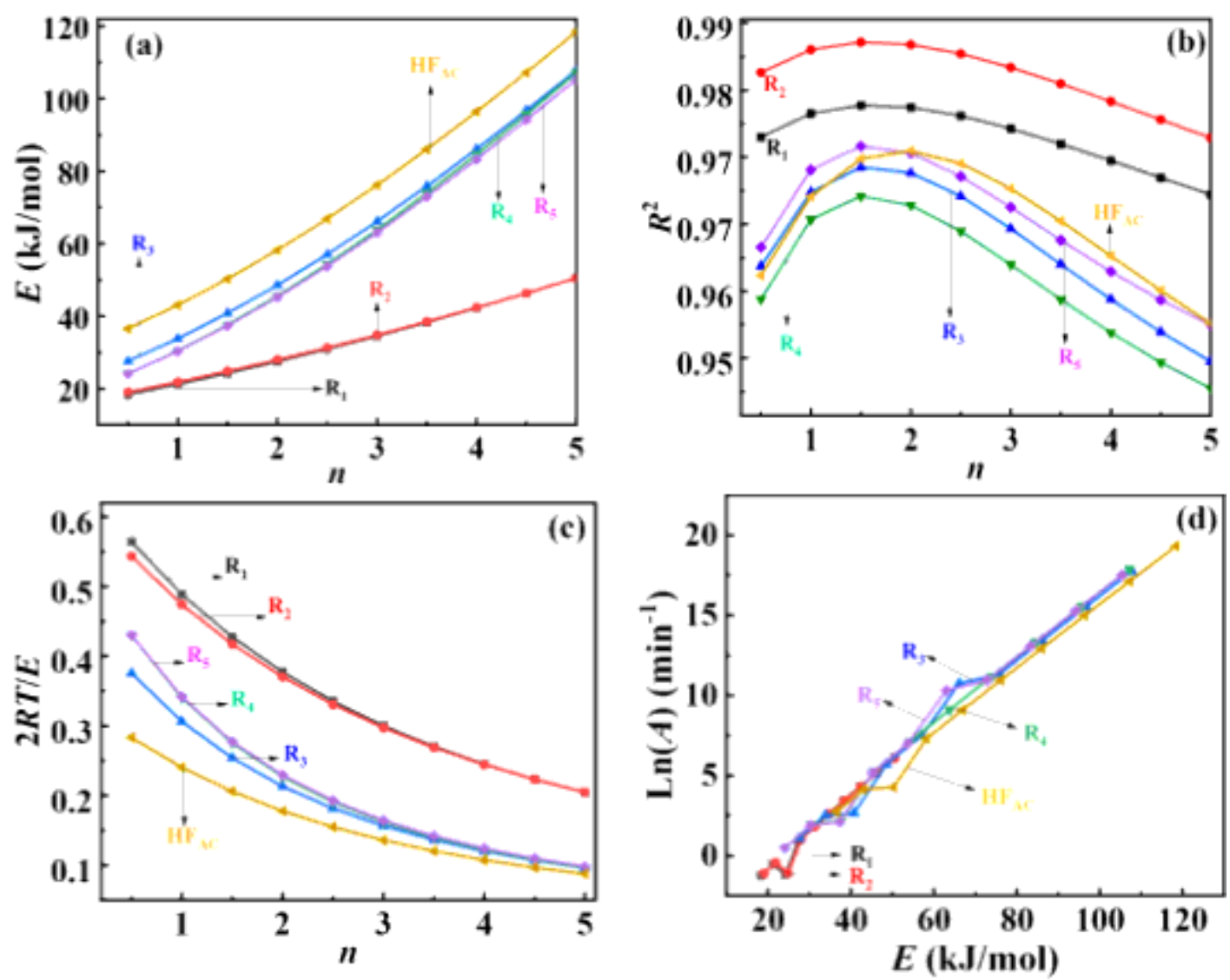

Figure 5

Relationship between kinetic parameters and reaction order. 